\title{
Pour une génétique de l'improvisation musicale (seconde partie)
}

Éléments méthodologiques et typologie de cas d'études

\section{Clément Canonne et Martin Guerpin}

\section{OpenEdition}

\section{Journals}

Édition électronique

URL : http://journals.openedition.org/genesis/4247

DOI : $10.4000 /$ genesis. 4247

ISSN : 2268-1590

Éditeur :

Presses universitaires de Paris Sorbonne (PUPS), Société internationale de génétique artistique littéraire et scientifique (SIGALES)

\section{Édition imprimée}

Date de publication : 1 juin 2019

Pagination : 169-182

ISSN : 1167-5101

\section{Référence électronique}

Clément Canonne et Martin Guerpin, « Pour une génétique de l'improvisation musicale (seconde partie) », Genesis [En ligne], 48 | 2019, mis en ligne le 01 juin 2020, consulté le 25 janvier 2021. URL http://journals.openedition.org/genesis/4247 ; DOI : https://doi.org/10.4000/genesis.4247 


\title{
Pour une génétique de l'improvisation musicale Seconde partie : Éléments méthodologiques et typologie de cas d'études*
}

\author{
Clément Canonne et Martin Guerpin
}

\section{Avant-textes et brouillons : documenter la genèse d'une improvisation}

\author{
Distinguer l'avant-texte et le brouillon \\ d'une improvisation
}

Selon Pierre-Marc de Biasi :

L'avant-texte désigne, dans le travail de l'écrivain, l'enchâ̂nement des opérations d'écriture qui ont précédé l'apparition du texte proprement dit. Le stade de l'avant-texte désigne donc celui du processus de production de l'œuvre, tel qu'il pourra être reconstitué par l'analyse des manuscrits de travail de l'auteur, puis interprété selon une méthode critique définie ${ }^{1}$.

Dans la genèse d'un texte, l'avant-texte inclut donc les documents préparatoires qui précèdent les premiers essais de rédaction : il rassemble les esquisses, les plans, les scénarios et les notes de documentation. Quant aux «brouillons», ils représentent un sous-ensemble de l'avant-texte que PierreMarc de Biasi qualifie d' «avant-texte rédactionnel». Maillon essentiel de la chaîne des transformations génétiques, le brouillon renvoie au «moment de textualisation qui constitue la médiation entre le projet de l'œuvre et le texte définitif $2^{\text {» }}$ et conduit de la première version rédigée d'un texte au texte publié.

Transposée au domaine de l'improvisation, la distinction entre avant-texte rédactionnel et non rédactionnel permet de distinguer plusieurs types de documents qui pourront figurer dans le dossier de genèse d'une performance improvisée. L'équivalent de l'avant-texte non rédactionnel, que l'on appellera phase de planification, inclut des documents produits par un musicien dans le but de préparer son improvisation : des schémas, de petits textes ou des indications musicales indiquant une gamme sur laquelle le musicien envisage de jouer à tel ou tel moment de son improvisation, ou encore une cellule mélodique ou rythmique qu'il désirerait exploiter. À ces documents peuvent s'ajouter des conversations ou des échanges de courriels entre les musiciens, ainsi que des entretiens de remise en situation avec le chercheur, effectués après une improvisation, permettant de mettre au jour le projet improvisatoire général auquel s'articule la performance considérée. Selon la manière dont les séances studio ont été enregistrées, les pratiques de conservation des archives enregistrées par les labels, mais aussi selon les types de pratiques improvisées et les habitudes particulières des musiciens, le nombre et la diversité de ces documents préparatoires peuvent varier sensiblement d'un dossier de genèse à l'autre. Un autre équivalent musical de l'avant-texte non rédactionnel mérite d'être mentionné : la partition ou le relevé noté d'un morceau. De tels documents étant rarement l'œuvre de l'improvisateur lui-même, leur présence dans un dossier de genèse a de quoi surprendre. Pourtant, ils peuvent conditionner son projet improvisatoire. Dans le domaine du jazz orchestral par exemple ${ }^{3}$, c'est la partition qui fixe la place d'une improvisation dans un morceau donné, sa longueur et la succession des accords dont elle doit tenir compte étant la plupart du temps fixées au préalable. Les autres documents à inclure dans le dossier de genèse d'une improvisation relèvent de ce qu'on appellera une phase de performance, correspondant au moment rédactionnel de l'avant-texte dans la génétique littéraire. Cette phase comporte les improvisations effectuées en amont de la

\footnotetext{
(*) La première partie de cet article, «Pour une génétique de l'improvisation musicale - Éléments théoriques », a été publiée dans le précédent numéro de la revue Genesis, n 47, p. 157-167.

1. Pierre-Marc de Biasi, «Qu'est-ce qu'un brouillon? Le cas Flaubert», dans M. Contat et D. Ferrer (dir.), Pourquoi la critique génétique? Méthodes et théories, coll. «Textes et Manuscrits», CNRS Éditions, 1998, p. 40.

2. Ibid., p. 31

3. Voir la fig. 1.b. de la première partie de l'article publié dans Genesis,
} $n^{\circ} 47$, p. 157. 
performance identifiée par le musicien, le producteur ou le chercheur, comme un point remarquable 4 - qu'il s'agisse des prises complètes (alternate takes) ou incomplètes (false starts) d'une session d'enregistrement en studio ou des improvisations «de répétition » qui se donnent précisément pour objectif de simuler les conditions du concert à venir.

La constitution du dossier de genèse d'une improvisation soulève deux difficultés. La première concerne la chronologie des documents de genèse relevant des phases de planification et de performance. Bien que les expressions laissent entendre que la première précède systématiquement la seconde, certains documents de planification peuvent être produits entre deux versions enregistrées ou deux improvisations «de répétition», c'est-à-dire pendant la phase de performance. C'est le cas, par exemple, lorsque l'improvisateur souhaite fixer des éléments qu'il entend conserver, ou replanifier son improvisation. L'enchevêtrement potentiel de ces deux phases a partie liée avec la dialectique, évoquée dans la première partie de cet article 5 , de l'instanciation et de la dérivation : les différentes manifestations d'un projet peuvent entraîner une modification de ce projet.

La seconde difficulté est elle aussi d'ordre chronologique. Comme dans le cas de la littérature, le généticien doit procéder à la mise en série chronologique des différents documents qui constitueront le dossier à partir duquel se déploiera son analyse. Cette mise en série peut s'avérer problématique dans le cas des enregistrements «historiques» réalisés par des labels discographiques privés. Un chercheur qui s'intéresse à la genèse de l'improvisation de Charlie Parker sur Relaxin'at Camarillo (Dial, 1947) devra d'abord regrouper les cinq versions enregistrées de ce morceau. Certaines peuvent être trouvées sur des disques publiées après la mort de Charlie Parker, et contenant des alternate takes. D'autres en revanche n'ont jamais été publiées et sont à rechercher dans les archives de Dial. Leur chronologie précise peut alors être établie grâce aux fiches de séances remplies par les producteurs et/ou les ingénieurs du son. La génétique de l'improvisation doit donc s'appuyer sur une science auxiliaire, la discographie, discipline constituée dans les années 1930 par des collectionneurs qui accordaient à la musique enregistrée un statut équivalent à celui de la musique notée 6 . La génétique de l'improvisation présente toutefois une spécificité : une partie du dossier de genèse, voire son intégralité, peut avoir été constituée par le chercheur lui-même, devenu le producteur de ses propres sources. C'est le cas lorsqu'il s'entretient directement avec les improvisateurs au sujet de leur projet improvisatoire. C'est le cas également quand il documente lui-même, au moyen d'un appareil enregistreur, des séances de répétition ou des concerts ne faisant pas l'objet d'une captation audio ou audiovisuelle.

À cette différence notable entre génétique littéraire et génétique de l'improvisation s'en ajoute une autre, liée elle aussi à l'ontologie de l'écriture et de l'enregistrement : celle du statut des brouillons écrits et des brouillons phonographiques.

\section{Du brouillon littéraire et du brouillon phonographique (logiques génétique et philologique, une distinction à revoir dans le domaine phonographique)}

\section{LA QUESTION DU STATUT DU BROUILLON}

Nous nous appuierons une fois encore sur les travaux de Pierre-Marc de Biasi, cette fois sur la distinction qu'il opère entre approche génétique et philologique. Alors que la génétique littéraire s'intéresse aux brouillons, c'est-à-dire à des documents que l'écrivain envisage comme potentiellement sujets à d'importants remaniements, la philologie étudie des «versions textuelles également définitives de la "même" œuvre [...] et qui peuvent revendiquer à chaque fois le statut de texte à part entière ${ }^{7}$. Dans le domaine de l'improvisation, cette distinction est bien moins nette. À la différence des brouillons littéraires, en effet, chaque version d'un projet improvisatoire est envisagée par le musicien comme une potentielle version définitive, qui peut prétendre au statut d'œuvre à part entière. Le travail du généticien de l'improvisation s'apparentera donc en partie à celui du philologue, en ce qu'il porte sur plusieurs performances possédant a priori un statut similaire.

4. Pour une définition des points remarquables, voir la première partie de notre article dans Genesis, ${ }^{\circ}$ 47, p. 157.

5. Genesis, $\mathrm{n}^{\circ} 47$, p. 164.

6. Michael Gray, «Discography: Discipline and Musical Ally», dans R. Green (dir.), Foundations in Music Bibliography, New York, The Haworth Press, 1993, p. 319-326.

7. Pierre-Marc de Biasi, «Qu'est-ce qu'un brouillon? Le cas Flaubert : essai de typologie...», op. cit., p. 43. 
L'une de ces performances peut toutefois être considérée comme un brouillon dans la mesure où le musicien en tient compte et la redéploie dans celle, postérieure, dont on se propose de retracer la genèse ${ }^{8}$. En ce sens, en dehors des très rares cas où une performance serait explicitement considérée par les musiciens comme une étape préparatoire à une «véritable» improvisation à venir (par exemple sous la forme d'un exercice spécifique, d'une «chauffe» ou d'une version «pour voir»), ce que nous appelons brouillon phonographique (que celui-ci résulte d'une session d'enregistrement en studio ou d'une documentation ethnographique) renvoie donc à une improvisation qui n'est pas envisagée en tant que brouillon en soi au moment de la performance. Elle ne le devient qu' a posteriori, de manière rétrospective. Dans cette perspective, une improvisation A peut être considérée comme un avant-texte de l'improvisation $\mathrm{B}$ dès lors que le musicien ou les musiciens réalisent l'improvisation $\mathrm{A}$ à partir de l'improvisation $\mathrm{B}$, ou en ayant présent à l'esprit l'improvisation $\mathrm{B}$, ou encore en faisant référence, d'une manière ou d'une autre, à l'improvisation B. Dans tous les cas, l'improvisation A est un avant-texte de l'improvisation B parce qu'elle entretient avec elle une relation intentionnelle. Cette différence pose une autre question essentielle : celle de la manière dont s'opère le passage d'une version à la suivante.

\section{LE PARADOXE DU BROUILLON IMPROVISATOIRE}

Grâce à la stabilité du support écrit, l'écrivain peut engendrer ses brouillons les uns à partir des autres par différentes opérations relevant de la manipulation directe de leur support matériel (ratures à même le manuscrit, dérivation d'un nouveau texte en ayant l'état précédent sous les yeux, etc.). Le temps de la genèse est donc pour lui un temps réversible. La situation est différente dans le cas de l'improvisation : chaque nouvelle réalisation d'un projet est à la fois un réinvestissement de ses versions précédentes et une performance entièrement nouvelle, produite selon une temporalité irréversible. Ici émerge ce que l'on pourrait appeler le paradoxe du brouillon improvisatoire : à chaque nouvelle réalisation d'un même projet, l'improvisateur recommence à zéro, même s'il ne part pas de rien. Comme le rappellent Lee Brown, David Goldblatt et Theodore Gracyk, alors que «la performance d'un work-in-progress implique que ce qui est joué le soit à nouveau, sous une forme affinée, dans le futur, chaque solo de saxophone de Parker est un nouveau commencement, et il importe peu de savoir s'il débouche ou non sur une autre performance ${ }^{9} »$. Le paradoxe du brouillon improvisatoire met donc en jeu les conditions de possibilité mêmes d'un processus de reprises et de corrections similaire à celui observable chez les écrivains. Elle implique également de réfléchir aux moyens dont dispose le généticien de l'improvisation pour observer ce processus.

\section{LA QUESTION DU PASSAGE D'UN BROUILLON À L'AUTRE}

Si le généticien de l'improvisation et le généticien littéraire peuvent tous deux s'appuyer sur les notes d'intention des artistes, ce dernier peut également retracer des opérations de dérivation matérielle en s'appuyant sur les ratures ou les ajouts effectués par l'écrivain, qui sont comme autant de signes matériels permettant d'observer le processus de création lié à l'écriture. Sauf exceptions, ces ratures ou ajouts n'ont pas d'équivalents dans le domaine de l'improvisation. Le généticien doit alors partir à la recherche d'opérations de dérivation entre des performances qui, à quelques exceptions près (par exemple les faux départs et les prises incomplètes issues des sessions d'enregistrement, ainsi que les étapes préparatoires évoquées plus haut), possèdent le même statut musical. Il peut alors s'appuyer sur deux critères :

- d'une part, un critère de proximité temporelle : l'opération de dérivation intentionnelle étant intégralement médiée par la mémoire de l'improvisateur, il semble davantage plausible de postuler l'existence d'une telle relation entre deux improvisations maximalement proches dans le temps (par exemple, entre la répétition du matin et le concert du soir ou entre deux prises successives d'un même morceau lors d'une session

8. En raison du statut particulier de l'improvisation enregistrée, il peut arriver que la version sélectionnée pour être publiée sur un album (la master take) ne soit pas la dernière version enregistrée, mais une version qui a pu servir de brouillon au cours de la séance d'enregistrement, avant d'être retenue comme la plus satisfaisante par le ou les improvisateurs.

9. Lee Brown, David Goldblatt et Theodore Gracyk, Jazz and the Philosophy of Art, New York, Routledge, 2018, p. 186. 
d'enregistrement) qu'entre deux improvisations séparées de plusieurs années 10 ;

- D'autre part, un critère d'accessibilité critique : on peut avoir de bonnes raisons de postuler l'existence d'une relation intentionnelle entre deux improvisations si l'on sait par ailleurs que la première improvisation a fait l'objet d'une réécoute critique voire d'une discussion entre les musiciens peu de temps avant la performance de la seconde improvisation.

Le processus de création qui intéresse le généticien de l'improvisation est donc conditionné par des médiations (la performance, la mémoire et la réécoute critique) sensiblement différentes de celles qui président au processus de création littéraire (l'écriture, la vue et la relecture critique). Cette différence de statut n'est pas sans incidence sur l'intentionnalité et sur la maîtrise du processus de création.

\section{LA QUESTION DE L'INTENTIONNALITÉ ET DE L'ORIENTATION DU PROCESSUS}

L'écriture permet à l'écrivain de conserver les parties du texte qui le satisfont. Elle lui laisse le temps de réfléchir aux modifications qu'il souhaite faire, mais aussi d'opérer ces modifications. Cette configuration ne fait évidemment pas du passage de l'avant-texte au texte un processus téléologique; mais elle permet de postuler le caractère intentionnel et maîtrisé de toutes les corrections apportées par l'écrivain à ses brouillons. Tel n'est pas le cas dans le domaine de l'improvisation puisqu'elle est soumise à des contraintes et à une urgence temporelles bien plus fortes que l'écriture. Le «paradoxe du brouillon improvisatoire» implique en effet qu'une nouvelle réalisation d'un projet donné puisse réinvestir des procédés déjà employés dans une réalisation précédente. En revanche, l'improvisateur ne peut rejouer de mémoire tous les passages qu'il aurait jugés satisfaisants dans cette réalisation. De plus, chaque nouvelle performance lui fait courir le risque de commettre des «erreurs» d'exécution qu'il n'avait pas faites dans les performances précédentes (d'où les false starts lors des séances d'enregistrement en studio) ou d'introduire dans son improvisation de nouvelles idées qui ne le satisferont pas. Dans ces conditions, le généticien ne peut considérer les différences constatées d'une performance à l'autre d'un même projet que comme le fruit d'une intentionnalité hypothétique (sauf dans les cas où il dispose des notes d'intention des musiciens). Le processus que le généticien de l'improvisation s'attache à retracer peut s'avérer extrêmement sinueux, autant sinon plus que les processus de création liés à l'écriture, qui ne sont ni téléologiques, ni même linéaires ${ }^{11}$. Alors que chaque nouvelle version d'un brouillon littéraire permet à l'écrivain de conserver tout ce qu'il avait validé et de ne modifier que ce qui ne le satisfaisait pas, la cö̈ncidence entre l'évaluation et son application est beaucoup plus incertaine dans le domaine de l'improvisation. Mais réciproquement, le processus de création à l'œuvre dans l'improvisation laisse plus de place à la sérendipité.

Une exception doit ici être mentionnée : celle des processus comprenant une phase de réenregistrement (re-recording), durant laquelle le musicien peut rejouer une partie de son improvisation, effacée au préalable par l'ingénieur du son. Dans ce cas, le musicien réentend au casque la musique précédemment enregistrée et ne joue qu' au moment où doit intervenir le complément. Ce cas, que l'on pourrait comparer à une correction de type effacement/remplacement sur un brouillon littéraire, demeure exceptionnel. Il en va de même pour tout le processus de post-production effectué par les ingénieurs du son, avec ou sans l'improvisateur, à partir d'une improvisation enregistrée et retenue pour faire l'objet d'une publicisation. Ces opérations peuvent être

10. Voici par exemple un extrait d'une conversation entre les improvisateurs du quintette «Umlaut» alors qu'ils s'apprêtaient à jouer ensemble pour la deuxième fois - leur premier concert en commun remontant à environ une année (Umlaut Quintet, répétition du 22 février 2016) :

«-A : Moi je serais assez pour développer un peu ce qu'on avait fait la dernière fois, enfin dans notre souvenir.

- S : Pour ma part, je n'en ai aucun souvenir!

- J : Surtout que c'était assez touffu, ça partait un peu dans tous les sens. - A : Moi je me souviens d'une sorte de drone bizarre...»

Si l'intervention de A semble nous inviter explicitement à lier ces deux improvisations, et donc à tenter de comprendre le déroulé de la seconde à la lumière de la première, il ressort en réalité clairement de la discussion que les musiciens ont un souvenir au mieux extrêmement vague de cette première improvisation. De plus, ils ne se souviennent pas exactement de la même chose. Dans cette perspective, il n'apparaît guère plausible de postuler l'existence d'une quelconque relation intentionnelle entre la seconde improvisation et la première.

11. Toute correction n'est pas nécessairement définitive, et tout nouveau brouillon n'efface pas nécessairement le précédent. L'on peut tout à fait envisager, d'un point de vue théorique et pragmatique, qu'un brouillon à l'état $n+1$ reprenne des passages présents dans l'état $n-1$ et qui avaient été supprimés ou modifiés dans l'état $n$. 
apparentées aux phases pré-éditoriales et éditoriales de la genèse d'un texte12. Elles s'étendent de la phase de mixage et de mastering jusqu'aux rééditions du vivant de l'auteur et aux rééditions posthumes. La plupart du temps, les improvisations concernées par ce travail ne sont modifiées qu'à la marge, sans que leur «texte» ne soit affecté. Il arrive en revanche que ces phases jouent un rôle décisif : c'est le cas par exemple de l'album Bitches Brew, enregistré en 1969 par Miles Davis et publié en 1970 par le label Columbia. La plupart des improvisations enregistrées lors des séances ont fait l'objet de multiples montages qui ont contribué de manière décisive à la forme qu'elles revêtent dans le disque publié. Les opérations réalisées lors de cette phase de postproduction mériteraient une étude particulière puisqu'elles concernent des processus de création différents de ceux qui sont au cœur de notre article.

Toutes ces précisions sur le statut de l'improvisation, sur les points de départ et d'arrivée possibles des processus qui président à sa réalisation et sur la nature des brouillons improvisatoires montrent que la génétique de l'improvisation ne peut se contenter de reprendre passivement les outils et les méthodes de la génétique littéraire. Moyennant les conditions et les aménagements qui ont été proposés et décrits, il apparaît néanmoins qu'une approche génétique peut légitimement être envisagée dans le domaine de l'improvisation musicale. Reste désormais à esquisser, à travers des cas d'études concrets, les différentes voies qu'elle est susceptible d'ouvrir à notre compréhension du travail de l'improvisateur.

\section{Esquisse d'une typologie des approches génétiques de l'improvisation musicale}

\section{Retracer des processus de création individuels : l'exemple de «Giant Steps » (John Coltrane)}

Un premier type de cas d'études concerne un improvisateur qui enregistre plusieurs versions d'un même projet au cours d'une séance en studio. La prise en compte de ce qui vient d'être joué est possible grâce à la mémoire du musicien et/ou à la réécoute critique d'une prise qu'il vient d'enregistrer. Le cas du saxophoniste John Coltrane enregistrant «Giant Steps» (Giant Steps, 1959), une composition de sa plume faisant une large place à l'improvisation, illustre parfaitement cette configuration ${ }^{13}$. Lors de deux séances organisées le 26 mars (8 prises) et le 5 mai 1959 (6 prises), quatorze versions intégrales ou incomplètes en ont été enregistrées sans répétition préalable. Lors de ces séances, les prises ont été soigneusement numérotées par l'ingénieur du son et le producteur. Leur chronologie peut donc être reconstituée de manière précise. La 13 e a finalement été retenue pour figurer dans l'album et constitue donc la master take, le point remarquable. De manière rétrospective, les prises précédentes prennent alors le nom d'alternate takes lorsqu'elles sont complètes, et de false starts lorsque l'enregistrement de l'improvisation a été interrompu. Dans ces conditions, les treize premières prises de «Giant Steps » peuvent légitimement être considérées comme des brouillons et rassemblées dans un dossier de genèse que vient compléter (ou en tout cas éclairer) la grille harmonique du morceau qui impose à l'improvisateur un ensemble de contraintes formelles, harmoniques et rythmiques.

Afin d'étudier les différentes prises de l'improvisation de John Coltrane, des transcriptions en ont été réalisées. Leur comparaison systématique permet de faire apparaître des opérations que la seule écoute ne permettrait pas de déceler ou, dans le cas contraire, de représenter et de communiquer à des lecteurs. Toutes les opérations ne peuvent certes pas être mises en évidence par la transcription en notation traditionnelle. Celle-ci n'en constitue pas moins un outil précieux pour le généticien ${ }^{14}$. La comparaison des transcriptions de ces treize prises, que l'on peut considérer comme treize versions de l' «improvisation de John Coltrane sur Giant Steps », fait apparaître plusieurs phénomènes remarquables.

Tout d'abord, le tempo adopté par Coltrane a évolué de 240 à 290 bpm (battements par minute) entre la première et la treizième version. Au fur et à mesure que le tempo augmente, le saxophoniste supprime progressivement les

12. Pierre-Marc de Biasi, «La critique génétique», dans D. Bergez (dir.), Méthodes critiques pour l'analyse littéraire, Paris, Bordas, 1990, p. 51-54. 13. Ce travail sur «Giant Steps» a fait l'objet d'une communication au colloque «Analyser les processus de création musicale» organisé à 1'Université de Montréal et à l'Université McGill en octobre 2013 (Martin Guerpin, «L'alternate take ou le jazzman au travail. Pour une approche génétique de l'improvisation »).

14. La notation ne permet en outre que de proposer une représentation simplifiée des hauteurs et des rythmes tels qu'ils sont réellement joués par l'improvisateur. 


\begin{tabular}{|l|l|}
\hline \multicolumn{2}{|c|}{26 mars 1959} \\
\hline Prise 1 & Incomplète \\
\hline Prise 2 & Faux départ \\
\hline Prise 3 & Incomplète \\
\hline Prise 4 & Incomplète \\
\hline Prise 5 & Alternate take \\
\hline Prise 6 & Faux départ \\
\hline Prise 7 & Incomplète \\
\hline Prise 8 & Alternate take \\
\hline & 5 mai 1959 \\
\hline Prise 1 (9) & Incomplète \\
\hline Prise 2 (10) & Incomplète \\
\hline Prise 3 (11) & Incomplète \\
\hline Prise 4 (12) & Faux départ \\
\hline Prise 5 (13) & Master take \\
\hline Prise 6 (14) & Alternate take \\
\hline
\end{tabular}

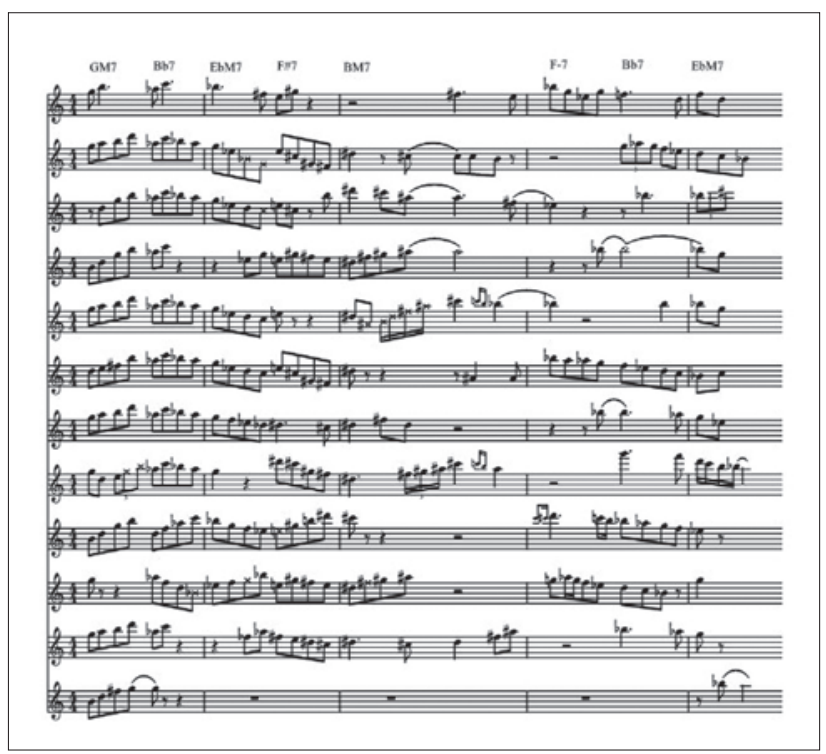

Fig. 1a (ci-contre) : Liste des prises de «Giant Steps» enregistré par John Coltrane.

\begin{tabular}{|ll|ll|l|ll|}
\hline BM7 & D7 & GM7 & Bb7 & EbM7 & Am7 & D7 \\
\hline GM7 & Bb7 & EbM7 & F\#7 & BM7 & Fm7 & Bb7 \\
\hline EbM7 & Am7 & D7 & GM7 & C\#m7 & F\#7 \\
\hline BM7 & Fm7 & Bb7 & EbM7 & C\#m7 & F\#7 \\
\hline
\end{tabular}

Fig. 1b (ci-dessus) : Grille harmonique de «Giant Steps».

Fig. 2 : Saturation rythmique de la grille de «Giant Steps» : points de départ (prise 1, choruses 1-12, fig. 2a, à gauche) et d'arrivée (prise 13, choruses 1-13, fig. 2b, à droite) du processus d'élimination des silences. Relevé et transcription : Martin Guerpin.

passages où il joue volontairement en arrière de la pulsation (laid back $)^{15}$, jusqu'à ne jouer que sur la pulsation. Cette accélération progressive s'explique sans doute par la difficulté de la grille harmonique du morceau. Caractérisée par des modulations systématiques par tierces majeures (en $s i$, en $s o l$ et en $m i$ bémol) qui se répètent dans un mouvement d'instabilité permanente, elle constitue littéralement un OVNI harmonique dans le paysage du jazz des années 1950. Le morceau n'ayant pas été répété au préalable par le quartet de John Coltrane, il est possible que les premières prises aient été réalisées dans un tempo assez lent pour laisser le temps aux musiciens de se familiariser avec les changements d'accords. Cette méthode consistant à jouer d'abord lentement un passage difficile puis de passer à un tempo progressivement plus important jusqu'à parvenir à la vitesse voulue est une pratique courante chez les musiciens.

L'autre trait caractéristique que révèle l'étude des différentes improvisations enregistrées par Coltrane est la présence d'un projet de «saturation de la grille harmonique». Prise après prise, en effet, les improvisations de Coltrane se font de plus en plus volubiles et laissent de moins en moins de place au silence (voir fig. 2). Dans la master take,

15. Il arrive fréquemment que les jazzmen décalent les attaques de leurs notes, ou ralentissent le débit de groupes de croches, afin de les déphaser très légèrement par rapport à la pulsation fixe donnée par la section rythmique. Cette pratique joue pour beaucoup dans l'effet de tension/détente rythmique caractéristique du swing. 

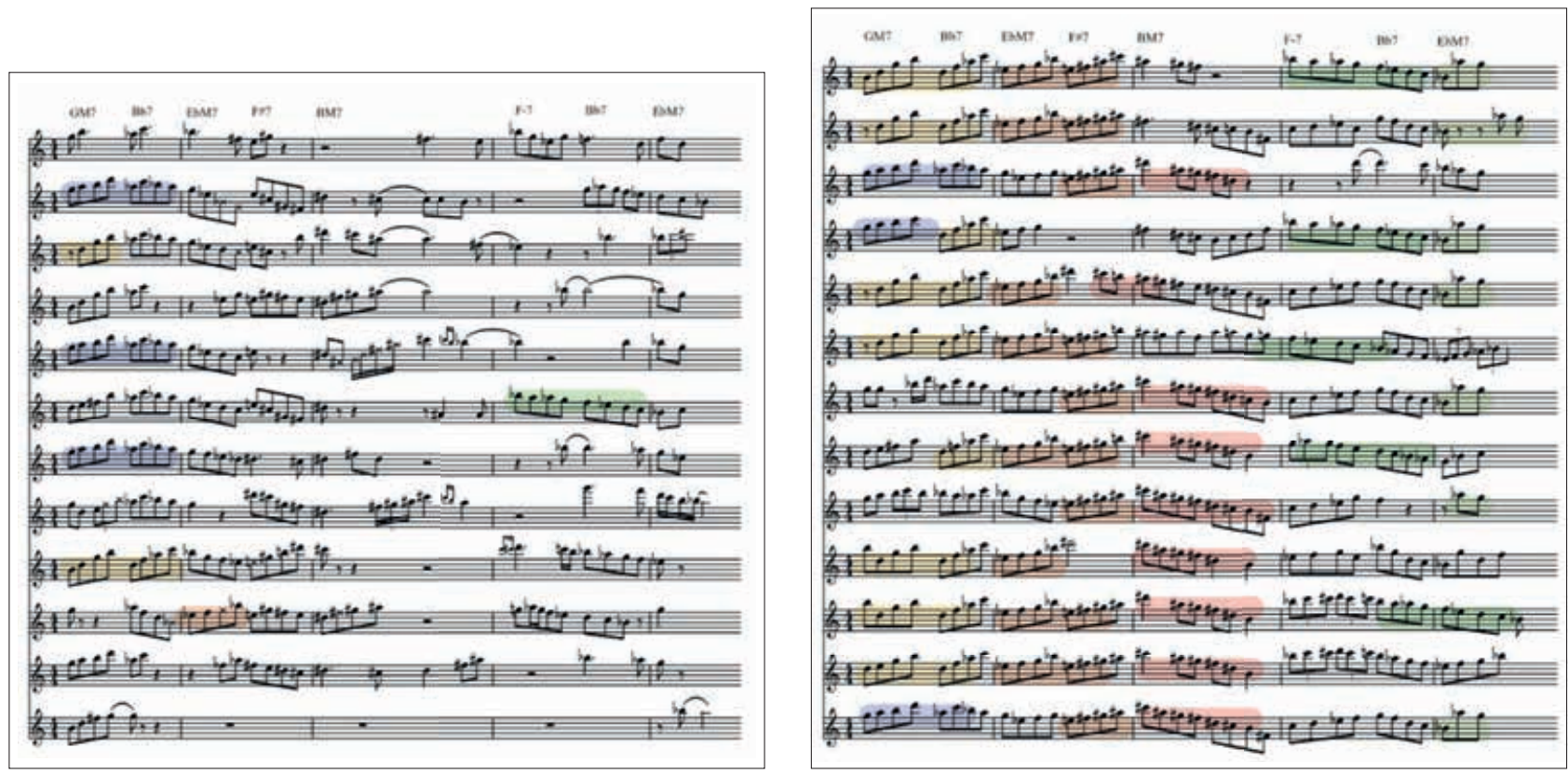

Fig. 3 : Le processus de sélection/reconduction et de suppression/renouvellement d'idées mélodiques dans les versions enregistrées de l'improvisation de John Coltrane sur «Giant Steps». Comparaison des cinq premières mesures des prises 1, choruses 1 à 12 (fig. 3a) et 13, choruses 1 à 13 (fig. 3b). Chaque couleur signale une idée reconduite par John Coltrane. Relevé et transcription : Martin Guerpin.

l'improvisation du saxophoniste consiste en un long flux ininterrompu de croches, ce qui relève d'un véritable tour de force au vu de la rapidité du tempo et de la complexité de la grille. La part du silence se trouve réduite au strict nécessaire : elle se limite aux moments où le saxophoniste reprend son souffle. Ce projet de saturation de la grille harmonique par des phrases en croches pourrait être considéré comme une stratégie au service de laquelle Coltrane recourt à différentes opérations tactiques qu'une analyse comparée des douze prises successives de «Giant Steps » permet d'identifier.

Les mesures 7 et 8 de la grille sont particulièrement intéressantes à cet égard. Elles font se succéder deux accords, BM7 et Fm7, qui présentent pour le saxophoniste une double difficulté. La première relève de l'ergonomie : au saxophone, la gamme de si majeur, qui est celle attendue lorsque l'on improvise sur un accord de BM7, nécessite des enchaînements de doigtés peu commodes. La seconde relève des habitudes harmoniques des jazzmen. Dans les années 1950, un enchaînement harmonique à haute vitesse tel que celui entre les deux accords évoqués, séparés par un triton, demeure exceptionnel. Dans les premières prises, John Coltrane choisit donc de terminer une phrase sur l'accord de BM7, de s'interrompre, puis de commencer une nouvelle phrase sur l'accord de Fm7. Cette tactique lui permet d'éviter de trouver un lien harmonique entre ces deux accords relativement éloignés. À partir de la prise $n^{\circ} 6$, toutefois, Coltrane commence à jouer des phrases qui s'étendent sur ces deux mesures, et qui correspondent parfaitement à l'enchaînement d'accords prescrit par la grille harmonique.

Une autre stratégie mise en place par Coltrane consiste à mémoriser et à incorporer, d'une prise à l'autre, des phrases correspondant à un enchaînement harmonique particulier. Certaines des phrases que l'on retrouve dans la master take sont ainsi présentes dès la première prise (voir fig. 3). D'autres, qui apparaissent dans les prises suivantes, se voient alors systématiquement réemployées. Ce processus de cristallisation mélodique ne concerne qu'un ensemble de phrases que l'analyse permet de circonscrire. D'autres, jugées insatisfaisantes ou simplement non mémorisées par le saxophoniste, n'apparaissent plus dès la deuxième prise, et sont remplacées par d'autres phrases.

La comparaison des différentes prises de «Giant Steps » met donc en évidence un processus très lisible de sélection/ reconduction et de suppression/renouvellement que l'on peut considérer comme un processus d'optimisation $16 \mathrm{du}$ projet de Coltrane pour ses improvisations sur «Giant Steps ». À ce titre, les douze premières versions enregistrées par le saxophoniste constituent un modèle de brouillons phonographiques que l'improvisateur met au service d'un projet aux caractéristiques aisément identifiables. L'approche des

16. Le terme d'optimisation nous semble plus adapté que celui d'amélioration car ce dernier met en jeu un jugement de valeur ou de goût qui n'est pas en question ici, et suggère que le processus de création analysé est un processus téléologique. 
processus individuels qui vient d'être présentée pourrait être élargie à d'autres pans de l'œuvre enregistré de Coltrane, qui regorge d'exemples potentiellement intéressants. «One Up, One Down », par exemple, a fait l'objet de six versions successives lors d'une même séance en studio, le 6 mars 196317. Le cas d' «Impressions » mériterait d'être également étudié puisque le saxophoniste l'a enregistré à 26 reprises au moins entre le 1er novembre 1961 et le 28 juillet 1965 , date du dernier enregistrement connu de ce morceau par le saxophoniste et son quartet ${ }^{18}$. Ce dernier type d'exemple permettrait de réinterroger les conditions de possibilité d'une étude génétique sur une temporalité plus longue que celle d'une séance d'enregistrement ou d'une tournée pendant laquelle les concerts se succéderaient quotidiennement ${ }^{19}$.

L'improvisation ne se réduit pas, toutefois, à des processus de création individuels. Elle se caractérise également par des interactions, certes plus ou moins marquées selon les pratiques, mais toujours présentes à l'esprit des improvisateurs. C'est pourquoi la génétique de l'improvisation doit tenir compte, autant que faire se peut, de la dimension collective des processus de création à l'œuvre dans l'improvisation.

\section{Retracer des processus collectifs : l'exemple de «Flamenco Sketches» (Miles Davis)}

Un exemple type de dossier de genèse permettant de faire apparaître la part de l'interaction dans la genèse d'une improvisation nous est fourni par le «Flamenco Sketches» enregistré par le sextette de Miles Davis (Kind of Blue, 1959) ${ }^{20}$. Six prises au moins de ce morceau ont été réalisées en l'espace de quelques minutes, lors d'une séance d'enregistrement organisée le 22 avril 195921. Seules la première et la sixième sont complètes. La dernière a finalement été retenue comme la master take. Outre ces prises, le dossier de genèse de l'œuvre comprend des commentaires de Miles Davis enregistrés après deux faux départs, et une feuille de papier à musique où le trompettiste a écrit les modes sur lesquels les musiciens devaient improviser.

Plus encore peut-être que dans «Giant Steps », la situation d'improvisation dans laquelle les musiciens sont placés lorsqu'ils jouent «Flamenco Sketches » doit être prise en compte par le généticien. Cette situation joue en effet un rôle déterminant dans la genèse du projet des improvisateurs : l'identité de Flamenco Sketches, en tant qu'œuvre, ne repose ni sur une mélodie particulière, ni sur une formule d'accompagnement caractéristique. Elle est assurée par la seule série de modes que Miles Davis a fait découvrir à ces musiciens au début de la séance du 22 avril. Le morceau consiste à répéter cette série de modes, sur laquelle chaque musicien peut improviser tour à tour. De plus, le nombre de mesures alloué à chacun de ces modes (le cadrage métrique de la grille harmonique) n'est pas fixé. Tout projet improvisatoire sur «Flamenco Sketches » doit donc s'organiser autour d'un impératif incontournable : la mise en place d'un système de coordination entre le soliste et la section rythmique (piano, contrebasse et batterie) qui l'accompagne. L'improvisateur devra en effet faire en sorte que ses collègues comprennent à quel moment moduler, mais aussi qu'ils modulent ensemble, de concert avec lui. L'analyse des différentes prises de ce morceau s'avère particulièrement intéressante car elle nous montre non seulement Miles Davis, mais aussi tout son groupe au travail. Elle permet de retracer quelques étapes de l'élaboration progressive d'un projet que l'on pourrait qualifier de «stratégie du silence».

Dans la première prise enregistrée de son improvisation sur «Flamenco Sketches», Miles Davis ménage de longs silences à la fin de ses phrases, parfois jusqu'à une mesure et demie. Ces silences sont systématiquement placés au moment des changements de mode. Ils permettent au trompettiste

17. Cette séance jusqu'à présent inédite a tout récemment été révélée au grand public (John Coltrane Quartet, Both Directions at Once: The Lost Album, enregistré le 6 mars 1963 à Englewood Cliffs, Impulse, 2018).

18. Ces données ont été produites à partir des travaux menés par le Jazz Discography Project Team < https://www.jazzdisco.org/john-coltrane/, consulté le 27 juillet 2018 >. Elles sont tributaires des connaissances actuelles sur la discographie de John Coltrane.

19. Pour une étude tentant de replacer le travail d'un groupe d'improvisation libre sur le long terme, voir Clément Canonne, «L'improvisation libre à l'épreuve du temps : logiques de travail et dynamiques créatives d'un duo d'improvisateurs », Revue de Musicologie, vol. 103, n 1, 2017, p. 137-167.

20. Ce travail sur «Flamenco Sketches» a fait l'objet d'une communication au colloque «Analyser les processus de création musicale» organisé à la Maison des Sciences Humaines de Lille en septembre-octobre 2011 (Martin Guerpin, «Jazzmen au travail. Essai d'analyse génétique des improvisations de Miles Davis sur Flamenco Sketches »).

21. Quatre sont actuellement disponibles dans différentes rééditions de l'album, mais dans l'avant-dernière, l'ingénieur du son annonce qu'il s'agit de la «prise $5 »$. 


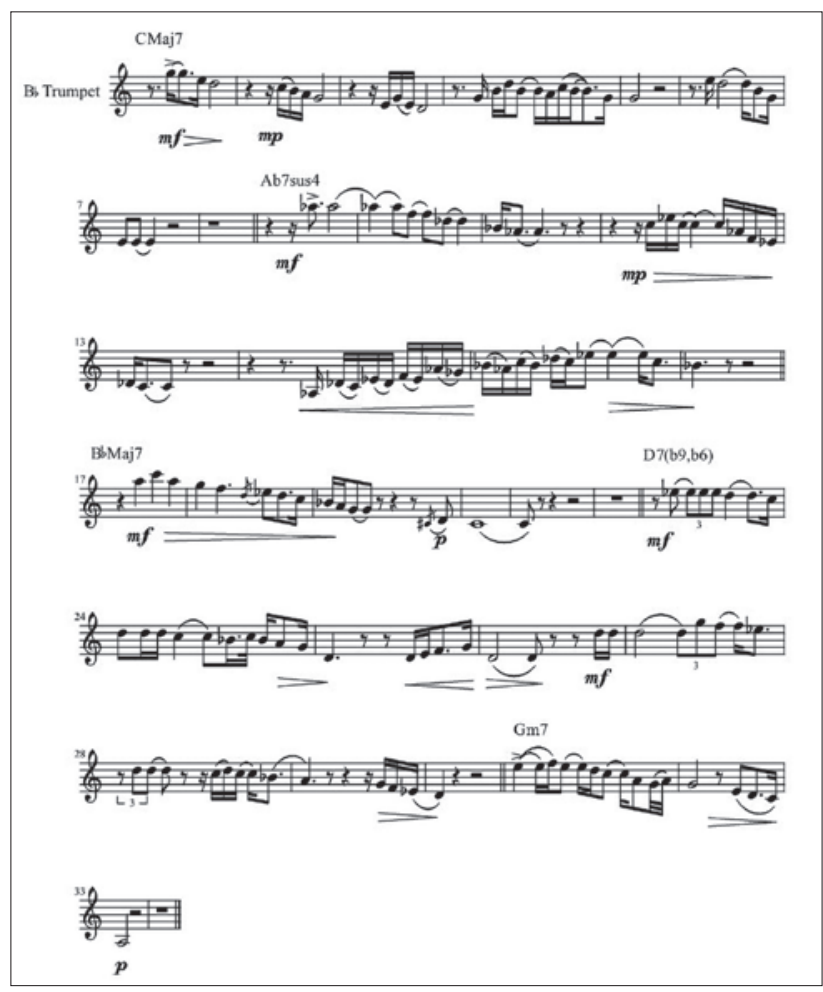

de laisser les trois musiciens de la section rythmique se coordonner entre eux pour moduler, minimisant ainsi les risques de conflit harmonique. Pareil conflit surviendrait en effet si Miles Davis changeait de mode au cours d'une phrase sans que la section rythmique ne puisse l'anticiper ou, réciproquement, si la section rythmique décidait de changer de mode alors que l'improvisateur continuerait de jouer sur le mode précédent. Cette stratégie semble planifiée dès la première prise puisque Miles Davis l'emploie lors des quatre changements de mode de la grille : terminer une phrase, laisser l'initiative du changement de mode à la section rythmique, et ne commencer une nouvelle phrase qu'une fois que le nouveau climat harmonique est installé.

Mais alors que cette stratégie relève de la précaution dans la première prise, Miles Davis l'adopte et la reconduit. Quelques minutes plus tard, en effet, lors de la sixième prise, le trompettiste et la section rythmique semblent avoir intégré l'usage du silence, au point de le réemployer systématiquement. Il devient dès lors possible à l'analyste de postuler une stratégie du silence, prisme à travers lequel il étudiera les différentes prises complètes et incomplètes de «Flamenco Sketches». D'abord utilisé de manière pragmatique comme une précaution, le silence est redéployé en tant que principe poétique, et devient par la même occasion un signal intelligible, convenu entre les musiciens. Outre la reconduction systématique de la stratégie du silence dans

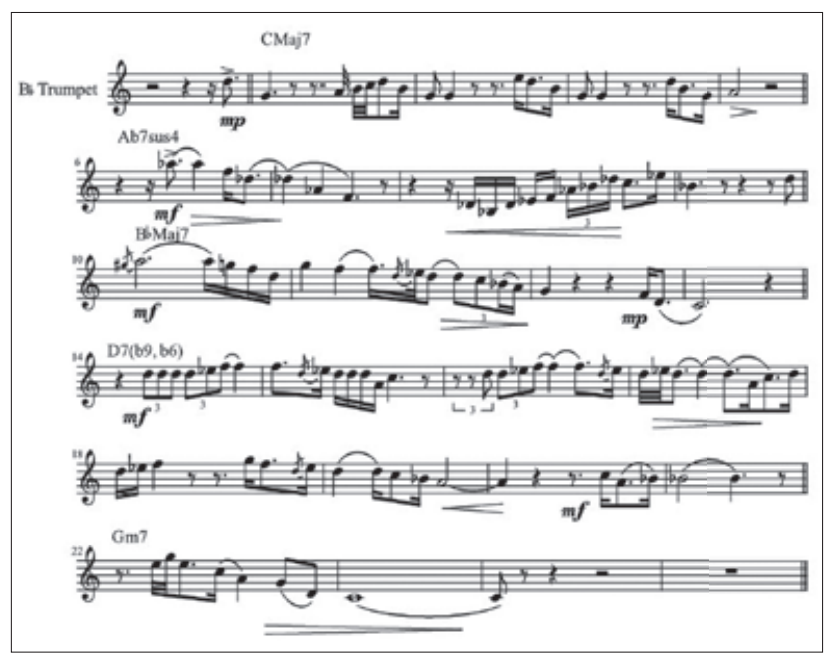

Fig. 4 : Prises 1 (fig. 4a, à gauche) et 6 (fig. 4b, à droite) de «Flamenco Sketches», improvisation de Miles Davis. Relevé et transcription : Martin Guerpin.

les différentes prises du morceau, cette évolution se traduit par un processus d'optimisation.

Après la première prise en effet, Miles Davis et la section rythmique abandonnent les longs silences de plus d'une mesure qui pouvaient traduire une hésitation, et passent plus rapidement à l'accord suivant. Cette assurance progressive dans la coordination des quatre musiciens peut être expliquée par la répétition d'un procédé déjà utilisé dans les prises précédentes, par le recours à des signes visuels que Miles Davis évoque à la fin de la cinquième prise ${ }^{22}$, mais aussi par un recours systématique à des carrures de quatre mesures.

Ce procédé permet de rendre le passage d'un accord à un autre plus prévisible pour la section rythmique car le répertoire du jazz dans les années 1950 fonctionne de manière presque exclusive sur des carrures de quatre mesures. Aussi les musiciens n'ont-ils à focaliser leur attention que sur les passages d'une carrure à une autre, plutôt que de

22. Cette prise incomplète et le dialogue qui s'ensuit font partie du dossier de genèse de l'improvisation. Les musiciens se sont interrompus suite à une mauvaise coordination lors du passage entre le premier et le second accord :

«- [Miles Davis:] You're not watching Bill.

- [Paul Chambers:] No, I'm sorry.

- [Miles Davis (à Irving Townsend, son producteur):] (We) try again Irving.

- [Irving Townsend:] Right. Six.» 


\begin{tabular}{|c|c|c|c|}
\cline { 2 - 4 } \multicolumn{1}{c|}{} & \multirow{2}{*}{ Accords } & \multicolumn{2}{c|}{ Nombre de mesures } \\
\cline { 2 - 4 } \multicolumn{1}{c|}{} & & Prise 1 (alternote take) & Prise 6 (master take) \\
\hline Introduction & $\mathrm{C} \Delta$ & 4 & 4 \\
\hline \multirow{4}{*}{ Miles Dawis } & $\mathrm{C} \Delta$ & 8 & 4 \\
\cline { 2 - 4 } & Absus4 & 4 & 4 \\
\cline { 2 - 4 } & $\mathrm{Bb} \Delta$ & 6 & 4 \\
\cline { 2 - 4 } & $\mathrm{D} 7(\mathrm{~b} 9, \mathrm{~b} 6)$ & 8 & 8 \\
\cline { 2 - 4 } & $\mathrm{G}-7$ & 4 & 4 \\
\hline
\end{tabular}

Fig. 5 : Nombre de mesures par accords dans «Flamenco sketches» (prises 1 et 6$)$.

se préparer à un éventuel changement de mode à chaque mesure. Les risques d'erreur s'en trouvent diminués : si un changement d'accord doit survenir, ce ne pourra être qu'après la quatrième mesure, ou après la huitième, mais pas après la troisième ou la sixième. C'est ainsi que, dans la master take, les modes de «Flamenco Sketches»se suivent de manière régulière, toutes les quatre mesures, ce qui réduit l'originalité potentielle du morceau, puisqu'il aurait pu se faire succéder par des segments de cinq ou sept mesures inusuels dans le jazz. Miles Davis ne rompt ce rythme harmonique que sur l'accord D79,6 (1'avant-dernier de la grille harmonique), sur lequel il s'attarde pendant huit mesures. Ce faisant, il met d'autant plus en valeur l'effet de tension créé par cet accord qui se révèle être une dominante lorsqu'il se résout sur celui de Gm. Ce réemploi optimisé de la stratégie du silence permet de considérer les cinq premières prises de «Flamenco Sketches» comme les brouillons phonographiques d'un projet de coordination et d'interaction entre les musiciens.

Les résultats qui viennent d'être présentés ne font qu'esquisser des travaux de plus grande envergure qui pourraient porter sur les alternate takes et/ou, à une autre échelle temporelle, sur les différentes versions d'un morceau contenant des improvisations enregistrées en concert par un musicien, au sein d'une même formation. Ces enregistrements réalisés en studio ou en concert offrent au chercheur un continent qui reste encore largement à explorer. Toutefois, les frontières de la génétique débordent les limites de ce continent : la possibilité offerte au chercheur de créer les sources de son investigation lui ouvre un champ de recherche potentiellement illimité. De fait, l'atelier du généticien ne se situe pas seulement dans les archives discographiques et dans le studio où il transcrit patiemment les enregistrements qu'il inclut dans un dossier de genèse. Il se trouve également sur le terrain où il enregistre les improvisations et/ou les commentaires des musiciens sur lesquels il souhaite travailler. Enquête phonographique par excellence, la génétique de l'improvisation peut se nourrir d'une approche ethnographique.

\section{De l'archive sonore au terrain ethnographique: les ateliers du généticien de l'improvisation}

Il s'agit alors pour le généticien d'enregistrer systématiquement les phases de planification et de performance précédant un point remarquable préalablement anticipé, par exemple en documentant l'intégralité d'une session d'enregistrement en studio censée aboutir à la production d'un nouvel album ${ }^{23}$, ou bien en étant présent aux séances de travail organisées par les musiciens en amont d'un concert. Un travail de ce type a été réalisé en documentant la «répétition » du quintette Umlaut (réunissant les cinq membres de la branche française du label du même nom - le saxophoniste Pierre-Antoine Badaroux, le contrebassiste Sébastien Beliah, le batteur Antonin Gerbal, la pianiste Ève Risser et le clarinettiste Joris Rühl) effectuée la veille de leur premier concert, le 5 juin 2015 à la Dynamo de Banlieues Bleues à Pantin - l'une des scènes les plus actives pour la diffusion du jazz et des musiques improvisées en Île-de-France. Nous disposons ainsi des enregistrements de l'improvisation (unique) réalisée lors de cette répétition et de l'improvisation effectuée en concert le lendemain, ouvrant ainsi la voie à une comparaison systématique des deux performances. Dans cette perspective, la documentation de l'intégralité des conversations entre les musiciens s'avère particulièrement précieuse pour préciser le type de relation pouvant unir la performance du concert à celle de la répétition.

Il faut d'abord signaler que, dans l'improvisation effectuée en répétition, les musiciens tentent de simuler

23. On trouvera une première tentative en ce sens dans l'article de Maya Gratier, Rebecca Evans et Ksenija Stevanovic, «Negotiations: Sound and Speech in the Making of a Studio Recording», dans E. Clarke et M. Doffman (dir.), Distributed Creativity: Collaboration and Improvisation in Contemporary Music, New York, Oxford University Press, 2018, p. $163-180$. 
les conditions du concert, que ce soit en termes d'instrumentation (le batteur part à la recherche d'un deuxième tom pour disposer d'un instrument comparable à celui qu'il utilisera le lendemain), de placement (les musiciens essayent différentes dispositions pour se rapprocher du plan de scène envisagé) ou de durée (en l'occurrence la durée typique d'un «set», soit une quarantaine de minutes). Cette improvisation est donc d'emblée appréhendée par les musiciens comme étant subordonnée au concert à venir, qui en est la raison d'être.

Ensuite, la répétition est l'occasion de voir émerger un projet improvisatoire, qui se dessine à la fois implicitement - au travers de l'improvisation réalisée à cette occasion - et plus explicitement, au travers des discussions suivant cette première improvisation. Celle-ci est en effet marquée par un goût certain pour les arrêts soudains et les passages de relais virtuoses d'un instrumentiste à l'autre (l'arrêt de l'un provoquant l'entrée de l'autre). Cet aspect est d'ailleurs clairement mentionné par la pianiste lors du «debriefing» de l'improvisation :

- E : Est-ce que vous ne pensez pas que la forme est générée par notre système d'interrupteurs, qu'on a beaucoup utilisé aussi ? Ça a vraiment généré une forme, ça. Ça nous a peutêtre empêché de faire des très longues parties qui se développent...

- $\mathrm{P}$ : Non parce que tu peux faire quelque chose qui se développe sans pour autant que ce soit une partie, avec des arrêts ou...

- E : Oui, oui, si c'est un peu mieux travaillé... le fait d'en parler, peut-être que ça va faire que ça va être mieux demain. Mais j'ai l'impression que c'était un mini-consensus de tout le monde, que c'était pour chacun de nous une clé, ces coupures un peu nettes... Ce qui est marrant, c'est qu'il y avait déjà des petits choix collectifs. S'il y en a qui détestaient la coupure d'un son net..

Il est intéressant de remarquer ici que, pour la pianiste, le fait d'expliciter les choix collectifs qui ont émergé spontanément de l'improvisation permet d'ouvrir la voie à un processus d'optimisation du projet improvisatoire « discontinuiste» qui semble (déjà) sous-tendre le travail de ce quintette.

Enfin, la répétition a également été le lieu d'un processus de sélection/élimination qui est resté, quant à lui, entièrement implicite, comme en témoigne cet extrait de conversation :
- J : Est-ce que vous voulez refaire un petit quelque chose?

$-\mathrm{P}$ : Moi je ne pense pas...

- J : Mais en l'état il va rester des choses en suspens...

- A : Moi je pense qu'on a travaillé en jouant, on a ouvert des portes, on a essayé de développer des trucs, de creuser...

$-\mathrm{P}$ : Non et puis je pense aussi que chacun sent bien ce qui marche, ce qui ne marche pas...

- J : Carrément !

La comparaison entre la répétition et le concert du lendemain permet néanmoins de préciser le contenu de ce processus. Par-delà la permanence du vocabulaire instrumental ou des matériaux sonores qui font partie du répertoire des musiciens et que l'on retrouve bien évidemment d'une performance à l'autre, par-delà la permanence des contraintes que fait peser le projet improvisatoire émergent (caractérisé par une esthétique de la discontinuité et le refus d'une approche «narrative» de l'improvisation) sur les discours individuels (les musiciens recourant à des sons tenus ou répétés, à des pseudo-boucles, à des gammes ou encore à des glissandi pour éviter toute structuration mélodique ou motivique), il est en effet remarquable de voir les musiciens préserver lors du concert un certain nombre de situations d'interaction explorées en répétition : on retrouve ainsi des passages marqués par une organisation duale du groupe (un sous-groupe produisant une texture pointilliste qui s'oppose aux sons continus d'un deuxième sous-groupe), des passages de polyphonie généralisée (dans lesquels chaque musicien développe son propre motif, ce qui donne l'impression d'une superposition de discours parallèles, en relation serrée les uns avec les autres), ou encore des passages de fusion harmonique ou spectrale qui donnent lieu à de longues séquences relativement statiques. Plus encore, ce sont des processus formels qui se voient conservés de la répétition au concert, comme le «système d'interrupteurs » mentionné par la pianiste ci-dessus, ou la condensation du matériau à laquelle se livre le groupe en passant d'un état liquide et continu (le glissando) à un état solide et discret (le déroulement de gammes ascendantes). Mais d'autres situations semblent au contraire avoir été écartées : nulle trace en effet dans le concert des imitations motiviques auxquelles s'étaient parfois adonnés la pianiste, le contrebassiste et le saxophoniste dans la première improvisation - ces passages, davantage évocateurs d'un jazz plus traditionnel, ne cadrant sans doute plus avec le 
projet improvisatoire ayant progressivement émergé lors de la répétition.

L'approche ethnographique permet donc d'enrichir considérablement la démarche comparative qui sous-tend la génétique de l'improvisation musicale. En effet, la documentation des conversations entre les musiciens (ou, le cas échéant, la réalisation d'entretiens de remise en situation avec les musiciens) vient en un sens pallier l'absence d'opérations matérielles (ratures, etc.), dont les brouillons sont habituellement le lieu, en guidant le généticien dans l'attribution de relations intentionnelles qui ne soient pas simplement hypothétiques.

Focalisation sur des phénomènes individuels ou collectifs, étude de processus observables sur une échelle de temps courte (les quelques heures d'une séance de studio) ou plus longue (les quelques jours d'une tournée de concert), recours à des sources préexistantes ou produites par le chercheur : on le voit, le spectre que peuvent couvrir les approches génétiques de l'improvisation est aussi varié que large. D'autant plus large qu'il ne se limite pas seulement au jazz et à l'improvisation libre. Le cadre théorique et méthodologique que nous avons tenté de définir pourra en effet être transposé à tout genre musical laissant une place à l'improvisation, comme la musique baroque ou encore le chaâbi algérien, par exemple.

\section{Conclusion}

Au terme de cette entreprise de cadrage théorique, méthodologique et programmatique de la génétique de l'improvisation, il est possible d'en formuler une première définition succincte. À équidistance des approches qui envisagent l'improvisation comme monade et comme work-inprogress, elle consiste en trois opérations critiques majeures :

- identifier et isoler des projets improvisatoires au sein de la production d'un musicien ou d'un groupe;

- constituer un dossier de genèse correspondant à chacun de ces projets;

- analyser les réemplois d'une version antérieure d'un projet dans une nouvelle version, jusqu'à celle définie comme un "point remarquable» par les musiciens ou le chercheur.

La génétique de l'improvisation se distingue donc des approches intertextuelles, qui ne tiennent pas compte de l'ordonnancement chronologique des différentes versions d'une improvisation. Elle se démarque également d'une stylistique de l'improvisation car elle ne porte pas sur l'ensemble de la production d'un improvisateur, ni même sur une période de sa production. Elle ne vise pas non plus à faire apparaître des traits récurrents qui constitueraient la spécificité de son langage. L'approche génétique privilégie l'étude des interactions entre la performance, l'écoute critique et la mémoire des musiciens. De ce fait, elle illustre parfaitement l'adage selon lequel tout artiste est aussi un critique. Elle permet également de faire apparaître, au sein de l'improvisation des processus compositionnels (sélection/ rejet du matériau, optimisation d'une stratégie qui peut servir d'élément unificateur d'une improvisation) déployés à partir d'un medium qui n'est ni l'écriture (comme c'est le cas dans la musique écrite), ni le son enregistré lui-même (comme c'est le cas dans les musiques électroacoustiques), mais la mémoire. Elle s'inscrit de ce fait dans un mouvement de remise en cause de l'opposition improvisation/composition déjà bien entamé depuis l'article fondateur de l'ethnomusicologue Bruno Nettl, «Thoughts on Improvisation» (1974), qui propose d'envisager le couple improvisation/ composition non pas comme une dichotomie mais comme les deux pôles d'un même continuum 24 .

Bien qu'elle envisage des documents de toute nature, la génétique de l'improvisation entretient une relation privilégiée avec l'enregistrement. Le plus souvent, en effet, les études génétiques sont fondées sur des matériaux enregistrés par des labels discographiques ou par le chercheur lui-même. Dans le premier cas en particulier, la recherche et/ou l'élaboration de ce matériau nécessite de connecter la génétique de l'improvisation à la recherche et à la science discographique. Le travail du généticien demeure en effet délicat en raison

24. Voir Bruno Nettl, «Thoughts on Improvisation: A Comparative Approach», The Musical Quaterly, vol. 60, n 1, 1974, p. 1-19. Sur la notion de «continuum de l'improvisation», voir également Bruce Ellis Benson, The Improvisation of Musical Dialogue: A Phenomenology of Music, Cambridge, Cambridge University Press, 2003.

25. Ces débats ont déjà cours dans le monde de la musique. La récente édition d'enregistrements inédits de Coltrane a par exemple soulevé des objections de la part de mélomanes refusant l'édition d'enregistrements non officiellement validés par les musiciens ou le leader des groupes qui en sont les auteurs (voir par exemple Denis Desassis, John Coltrane Quartet. Both Directions at Once: The Lost Album < https://www.citizenjazz.com/John-Coltrane-3475981.html>, consulté le 2 août 2018. 
de l'absence d'éditions discographiques reprenant la totalité des versions intégrales ou non enregistrées en studio et incluant tous les autres documents de genèse susceptibles d'avoir été enregistrés et/ou conservés. En l'état actuel des publications dans ce domaine, il manque donc au généticien des sources auxquelles s'abreuver. L'une des perspectives ouvertes à la génétique de l'improvisation réside donc dans la réalisation d'éditions génétiques, dont le modèle pourrait s'inspirer de celles consacrées par des chercheurs de l'ITEM aux manuscrits d'écrivains. De telles éditions pourraient donner lieu à de nouvelles réflexions sur la nature et le statut de l'œuvre enregistrée d'un musicien ou d'un groupe 25 . En même temps que la génétique de l'improvisation ouvre au chercheur un vaste champ d'investigation susceptible d'enrichir notre compréhension du travail de l'improvisateur, elle dévoile donc au mélomane un immense réservoir de matériaux encore inédits ou peu connus.

Mais au-delà, ou plutôt en deçà de cette perspective, l'une des principales ambitions de la génétique de l'improvisation consiste à faire apparaître une typologie d'attitudes des musiciens face à l'improvisation, de l'impératif de non-répétition observable chez des musiciens tels que Lee Konitz ou Derek Bailey, à la reprise entêtée de certains procédés ou de certains scénarios, chez John Coltrane ou le quintette Hubbub - un groupe d'improvisation «libre» à l'identité esthétique particulièrement bien caractérisée.
À terme, le développement d'études de cas permettra de tester certaines hypothèses comme celle, intellectuellement séduisante, d'une correspondance entre cette typologie et celle des pratiques artistiques mettant en jeu l'improvisation (théâtre, slam, performances, etc.). Dans le domaine de la musique, elle pourrait s'appliquer à l'ensemble des pratiques liées à l'interprétation, du jazz aux musiques improvisées en passant par celles des musiciens baroques. Les travaux menés dans ce sens pourront ensuite être articulés avec ceux qui, dans le domaine de la littérature, laissent à penser que l'usage des brouillons varie sensiblement d'un écrivain et d'un genre à l'autre26.

Enfin, l'élargissement potentiel de la génétique de l'improvisation à d'autres genres musicaux laisse entrevoir la possibilité d'une nouvelle extension du domaine de la génétique, qui viendrait prolonger celles évoquées à l'orée de cet article. La génétique de l'improvisation que nous avons présentée pourrait en effet faire l'objet d'une généralisation à l'ensemble des pratiques liées à l'interprétation et à la performance d'œuvres de tradition écrite ou orale, moyennant des aménagements conceptuels et méthodologiques dont la description nécessiterait un autre article.

26. Pierre-Marc de Biasi, «Qu'est-ce qu'un brouillon? Le cas Flaubert », op. cit., p. 32 .

MARTIN GueRPIN est agrégé de musique, lauréat du prix Socan-Proctor (2013) et du prix de la Société québécoise de recherche en musicologie (2013). Il prépare un doctorat sur les «Enjeux esthétiques de la réception du jazz dans le monde musical savant parisien de l'entre-deux-guerres ». Ses recherches portent également sur les processus de création à l'œuvre dans l'improvisation. Ses travaux ont fait l'objet de publications dans les Cahiers de la SQRM (2014) et dans Eurojazzland. Il enseigne au Conservatoire de Paris et à l'Institut d'études politiques de Paris. Depuis 2014, il fait partie du comité directeur des Cahiers du Jazz. Saxophoniste, il se produit régulièrement avec le Gil Evans Paris Workshop de Laurent Cugny.

martin.guerpin@gmail.com

CLÉment CANONnE est chargé de recherche au CNRS, rattaché à l'équipe Analyse des pratiques musicales au sein de l'UMR 9912 «Sciences et technologies de la musique et du son» (IRCAM-CNRS-Sorbonne Université). Ses recherches portent principalement sur la question de l'improvisation, envisagée à la fois comme pratique et comme paradigme. Son travail récent a fait l'objet de publications dans plusieurs revues internationales (Cognition, Music Theory Online, Revue de musicologie, Psychology of Music, etc.). Il s'intéresse également à la philosophie de la musique, a dirigé un ouvrage collectif : Perspectives philosophiques sur les musiques actuelles (Delatour, 2017) et a traduit et introduit, en collaboration avec Pierre Saint-Germier, une sélection des Essais de philosophie de la musique de Jerrold Levinson (Vrin, 2015).

clementcanonne@hotmail.com 


\section{Pour une génétique de l'improvisation musicale}

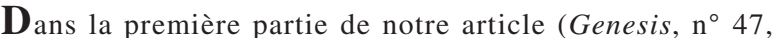
p. 155-167), à dominante théorique, nous avons identifié les conditions de possibilité d'une génétique de l'improvisation musicale, introduit les notions de projets improvisatoires et la dialectique de l'instanciation et de la dérivation qui caractérise la dynamique de la relation génétique. La seconde partie de l'article se concentre sur des questions de méthodologie et présente des premiers cas d'études. Nous abordons tout d'abord les conditions de possibilité d'un transfert des méthodes et des outils de la génétique littéraire au domaine de l'improvisation musicale. Après avoir introduit la notion de «brouillon phonographique», nous présentons quelques applications concrètes de l'analyse génétique de l'improvisation, en faisant émerger, dans une perspective programmatique, une typologie de cas permettant d'aborder différentes problématiques afférentes aux processus de création à l'œuvre dans l'improvisation.

In the first part of our article (Genesis, $\mathrm{n}^{\circ} 47$, p. 155-167), which is mainly theoretical, we have identified the conditions under which a Genetics of musical improvisation is possible. In doing so, we have introduced the concept of improvisational project and unpacked the dialectics of instantiation and derivation that characterizes the dynamics of improvised performances over time. The second part of the article focuses on methodological issues and presents representative case studies. We first discuss the extent to which methods and tools drawn from literary genetics can make sense in the field of musical improvisation. After having introduced the notion of phonographic draft, we apply it to a range of concrete examples and show how it can be used for a genetic analysis of improvisation. Given the programmatic dimension of this article, these examples are presented as a typology of case studies that sheds new lights on different issues at work in the creative process of improvisation.

Im ersten Teil unseres Artikels (Genesis, Nr. 47, S. 155-167), der hauptsächlich theoretischer Natur ist, haben wir die Bedingungen einer möglichen Genetik der musikalischen Improvisation herausgearbeitet und haben die Konzepte der „Improvisationsprojekte“ und die Dialektik von Instantiierung und Ableitung eingeführt, welche die Dynamik der genetischen Beziehung charakterisiert. Der zweite Teil unseres Artikels konzentriert sich auf methodologische Fragen und präsentiert erste Fallstudien. Wir diskutieren zunächst die Bedingungen für die Möglichkeit einer Übertragung von Methoden und Werkzeugen der literarischen Genetik auf das Gebiet der musikalischen Improvisation. Nachdem wir den Begriff des ,phonographischen Entwurfs“ eingeführt haben, stellen wir einige konkrete Anwendungen der genetischen Analyse der Improvisation vor, indem wir aus programmatischer Sicht eine Fall-Typologie herausstellen, die es ermöglicht, verschiedene
Fragen im Zusammenhang mit dem improvisierenden Schaffensprozess zu erörtern.

En la primera parte de nuestro artículo (Genesis, n 47, p. 155-167), predominantemente teórico, habíamos identificado las condiciones de posibilidad de una genética de la improvisación musical e introducido las nociones de proyectos improvisatorios y la dialéctica de la instanciación y de la derivación que caracteriza la dinámica de la relación genética. La segunda parte del artículo se centra en algunas cuestiones metodológicas y presenta los primeros casos de estudios. Primeramente, reflexionamos acerca de las condiciones de posibilidad de una transferencia de los métodos y las herramientas de la genética literaria al campo de la improvisación musical. Después de haber introducido la noción de "borrador fonográfico", presentamos algunas aplicaciones concretas del análisis genético de la improvisación, poniendo de manifiesto, con una perspectiva programática, una tipología de casos que permiten abordar diferentes problemáticas relativas a los procesos de creación presentes en la improvisación.

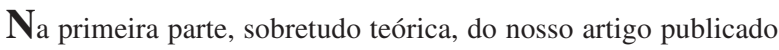
em Genesis, n 47, p. 155-167, identificámos as condições em que se pode observar uma genética da improvisação musical, introduzimos as noções de projecto improvisador e a dialéctica da instanciação e da derivação, que caracterizam a dinâmica da relação genética. A segunda parte do artigo concentra-se em questões de metodologia e apresenta os primeiros estudos de caso. Primeiro, discutimos a viabilidade de uma transferência de métodos e ferramentas da genética literária para o campo da improvisação musical. A partir da noção de «rascunho fonográfico», apresentamos aplicações da análise genética da improvisação, de que emerge, numa perspectiva programática, uma tipologia de casos e processos de criação que intervêm na improvisação.

Nella prima parte del nostro articolo (Genesis, $n^{\circ}$ 47, p. 155-167), d'impianto teorico, avevamo identificato le condizioni di possibilità di una genetica dell'improvvisazione musicale, introducendo le nozioni di "progetti improvvisatori" e la dialettica dell'istanziazione e della derivazione, che caratterizza la dinamica della relazione genetica. La seconda parte dell' articolo si concentra su questioni metodologiche e presenta i primi studi analitici. Vi si affrontano anzitutto le condizioni di possibilità di un trasferimento di metodi e strumenti dalla genetica letteraria al campo dell'improvvisazione musicale. Dopo aver introdotto la nozione di "scartafaccio fonografico", si presenta in seguito qualche applicazione concreta dell'analisi genetica dell'improvvisazione, facendo emergere, in un'ottica programmatica, una tipologia di casi che permettono di affrontare diverse problematiche riguardanti il processo di creazione che opera nell'improvvisazione. 